\title{
Internationale Studienergebnisse
}

\section{VISUELLE WAHRNEHMUNG}

\section{FEW-2 begrenzt aussagekräftig, aber konkurrenzlos}

Kinder zwischen vier und sechs Jahren haben häufig Schwierigkeiten, den Entwicklungstest der visuellen Wahrnehmung (FEW-2) erfolgreich zu bewältigen. Aufgrund man-

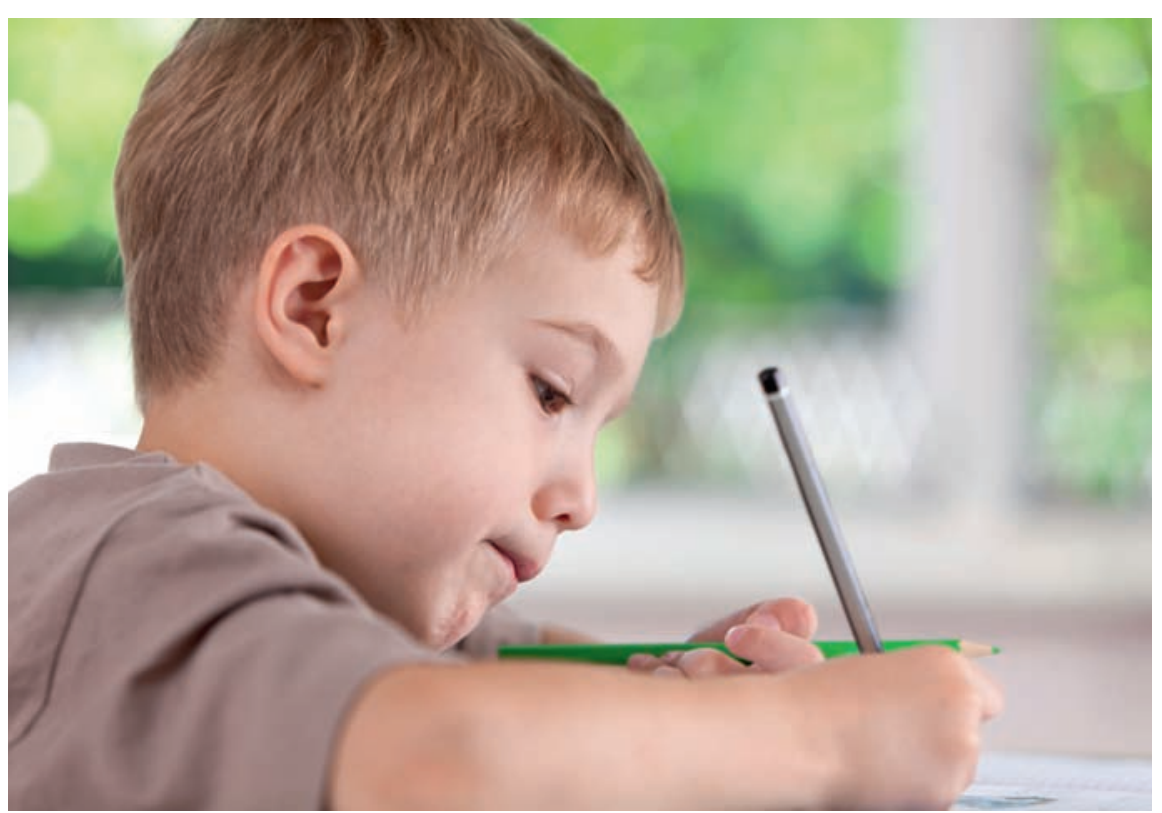

gelnder Alternativen stellt er dennoch das brauchbarste Verfahren dar, um die visuellräumlichen Leistungen zu überprüfen. Zu diesem Schluss gelangte eine Forschungsgruppe um die Ergotherapeutin Peggy Beuthan an der Privaten Universität für Gesundheitswissenschaften, Medizinische Informatik und Technik Hall in Österreich.

In einer experimentellen Studie untersuchten die Forscher mithilfe von 40 Mädchen und 40 Jungen im Alter von vier bis sechs Jahren, wie reliabel der FEW-2 und seine Subtests bei dieser Altersgruppe sind. Im Gegensatz zu Untersuchungen aus dem Jahr 2008 erreichte der Test deutlich geringere Reliabilitätswerte $(\alpha=.67)$ als empfohlen $(\alpha=.90)$. Dabei schnitt er bei den jüngeren Kindern etwas besser ab als bei den älteren.

Trotz des ernüchternden Ergebnisses empfehlen die Forscher, den FEW-2 weiterhin bei dieser Altersgruppe anzuwenden. Sie betrachten ihn als praktikables Verfahren, um gleichzeitig die motorikabhängige und motorikfreie visuell-räumliche Wahrnehmung zu testen. Allerdings fordern sie, die Zuverlässigkeit des FEW-2 anhand größerer Stichproben zu überprüfen.

$a k b$ ergoscience 2011; 6: 12-22

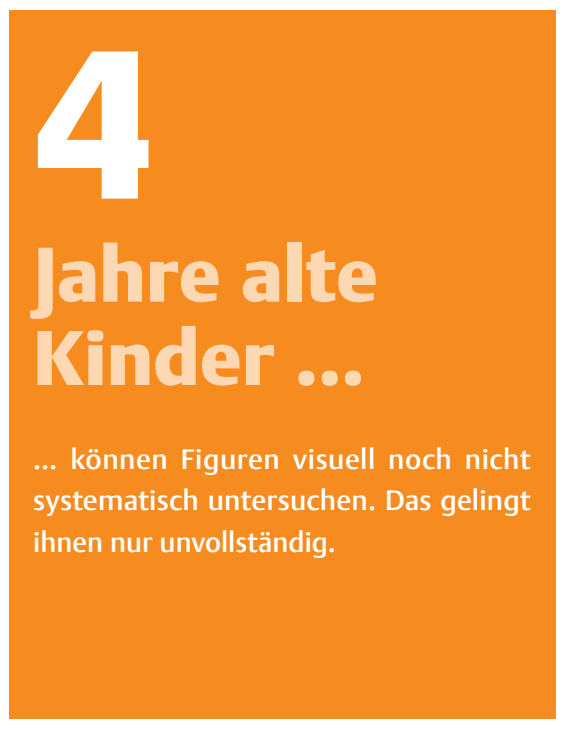

FEW-2

\section{Visuelle Wahrnehmung testen}

Frostigs Entwicklungstest der visuellen Wahrnehmung - 2 (FEW-2) ist die deutsche Adaptation des Developmental Test of Visual Perception - 2 (DTVP-2).

Die beiden Instrumente ermöglichen die diagnostische Differenzierung visueller Wahrnehmung und gehen auf die Lehrerin und Psychologin Marianne Frostig zurück. Ihre Subtests gliedern sich in die Bereiche Auge-Hand-Koordination, Lage im Raum, Abzeichnen, Figur-Grund, räumliche Beziehungen, Gestaltschließen, visuomotorische Geschwindigkeit und Formkonstanz. 
BRETTSPIEL „SEXUALITÄT UND CHRONISCHE KRANKHEITEN“

\section{Let's talk about sex}

Das niederländische Brettspiel „SeCZ TaLK“ (Seksualiteit en chronische Ziekten, zu Deutsch: Sexualität und chronische Krankheiten) kann chronisch erkrankte junge Menschen dazu animieren, sich über Sexualität, persönliche Beziehungen und Zukunftsvorstellungen auszutauschen. Ein Forscherteam um die Sozialwissenschaftlerin Heleen Hogeschool Rotterdam, Niederlande.

„SeCZ TaLK“ besteht aus einem Spielbrett und 66 Karten und findet in Kleingruppen von drei bis acht Teilnehmern statt. Es erfordert keine spezielle Vorbereitung und eignet sich für verschiedene Altersgruppen und Institutionen. Die Forscher implementierten das Spiel in fünf verschiedenen Einrichtungen. Anschließend befragten sie 85 chronisch erkrankte junge Menschen zu dem Spiel. Diese litten unter anderem an neuromuskulären Problemen, HIV, motorischen Einschränkungen und waren zwischen 11 und 28 Jahre alt waren. Die Mehrheit von ihnen sowie die zusätzlich befragten 12 Betreuungspersonen schätzten es als wichtig ein, sich über Sexualität auszutauschen, und fühlten sich durch das Spiel angeregt, sich van der Stege entwickelte das Spiel an der frei zu äußern. Dabei stieß das Brettspiel bei den weiblichen Teilnehmern und Jugendlichen unter 15 Jahren auf die höchste Resonanz.

Damit eine angenehme und offene Gesprächsatmosphäre entstehen kann, geben die Forscher zukünftigen Anwendern zu bedenken, die Gruppen homogen zusammenzusetzen.

Saja

WtvE 2011; 1: 21-37

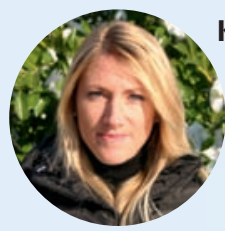

Kommentar > Spielerisch solche Studien sind in unserer Forschungslandschaft herzlich willkommen. Vor allem, wenn das Forschungsergebnis aus einem Spiel besteht, mit dem man direkt arbeiten kann. Besser können sich Theorie und Praxis kaum ergänzen.

Bemerkenswert, dass sich gerade die jungen Studienteilnehmer so positiv zu „SeCZ TaLK“ geäußert haben, fühlt sich doch gerade die über Sexualität sprechen -
Altersgruppe der unter 15-Jährigen häufig unsicher, wenn es um Sexualität geht. Offensichtlich hat das Spiel das Potenzial, diese Hemmschwelle zu überwinden.

Schade, dass „SeCZ TaLK“ bislang lediglich im niederländischen Sprachraum erhältlich ist. Das Thema bietet sich geradezu für eine Abschlussarbeit an: übersetzen, validieren und erproben. Das Ergebnis wäre sicherlich eine Bereicherung für den deutschen Sprachraum.

Simone Gritsch, Ergotherapeutin BcOT (NL)

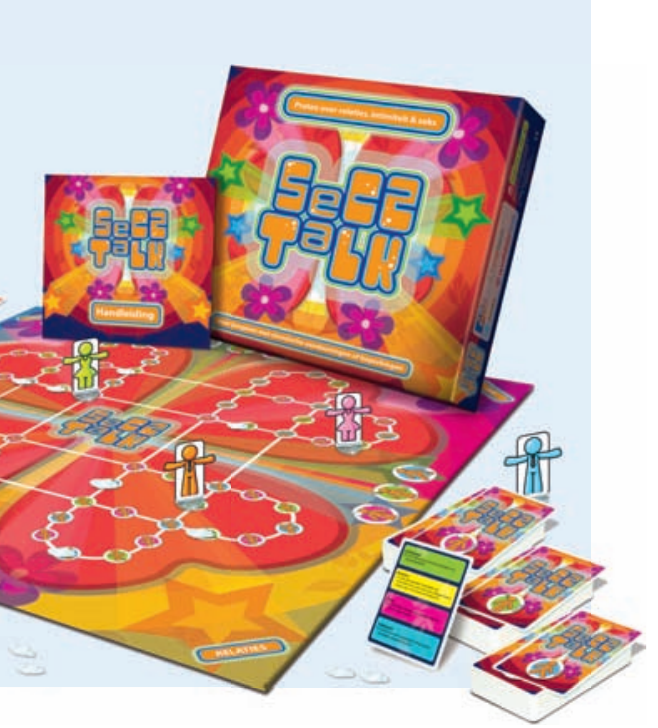

\section{AUFKLÄRUNG}

\section{4- bis 17-Jährige in Deutschland}

sind sich nicht sicher, ob sie aufgeklärt sind

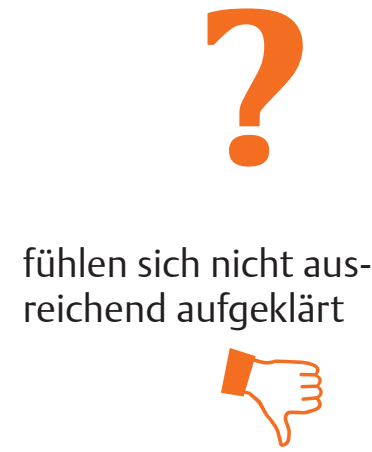

$12 \%$ fühlen sich aufgeklärt

$84 \%$

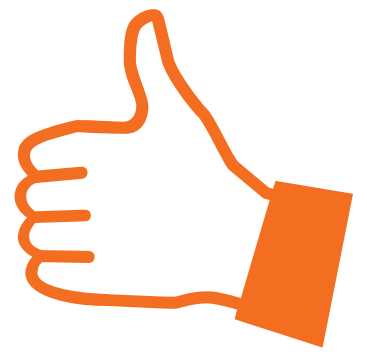
lichen ...

i. in Deutschland verhüten pflichtbewusst beim ersten Mal. Europaweit sieht es da ganz anders aus: Fast jeder Zweite hat ungeschützten Sex mit einem neuen Partner. In Thailand verhüten die Jugendlichen am wenigsten. 


\section{Wissenschaft}

PRÄVENTION IM ALTER

\section{Ältere Menschen profitieren von Ergotherapie}

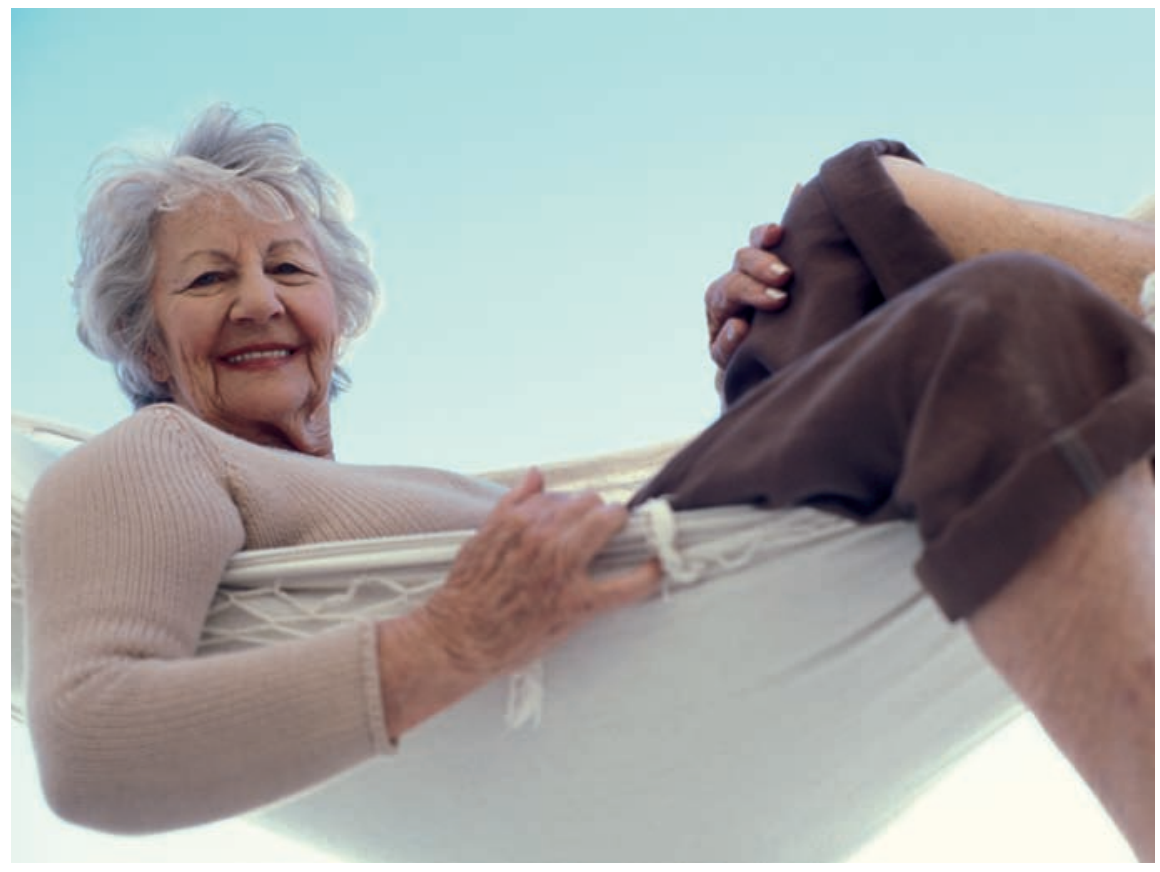

Ergotherapeutische Präventionsmaßnahmen, die auf verschiedene Lebensbereiche abzielen, fördern die Gesundheit und das seelische Wohlbefinden älterer Menschen. Das besagt die umfassende Studie eines Forschungsteams um Dr. Florence Clark aus der
Abteilung für ergotherapeutische Wissenschaft und Therapie an der Zahnheilkundeschule in Los Angeles, USA.

Insgesamt nahmen 460 Menschen, die zwischen 60 und 95 Jahre alt waren, an der randomisierten kontrollierten Studie teil. Im
Gegensatz zur Kontrollgruppe beteiligten sich die Probanden der Interventionsgruppe sechs Monate lang an diversen präventiven Einzelund Gruppeninterventionen - sowohl im therapeutischen Setting als auch im häuslichen und gemeindenahen Umfeld. Dazu gehörte es einerseits, Veränderungswünsche aufzudecken, und andererseits, Möglichkeiten zu finden, diese im alltäglichen Leben umzusetzen und zu integrieren. Die Probanden beider Gruppen durchliefen diverse Assessments. Auf diese Weise konnten die Forscher feststellen, ob sich die Lebenszufriedenheit und die kognitiven Fähigkeiten der Senioren während der sechs Monate verändert hatten.

Die Ergebnisse zeigen, dass sich die kognitiven Fähigkeiten der Probanden nicht verbessert hatten. Die ergotherapeutischen Interventionen hatten sich aber besonders positiv auf ihr seelisches Wohlbefinden ausgewirkt. Zudem konnten die Forscher nachweisen, dass sich Präventionsmaßnahmen wie diese kosteneffektiv auswirken, weil sich beispielsweise das Schmerzerleben der Teilnehmer reduzierte und sie so länger gesund blieben.

dawo

JECH 2011; doi: 10.1136/jech.2009.099754

\section{ZITAT}

"Das große Ziel des Lebens ist nicht Wissen, sondern Handeln."

Thomas Huxley (1825-1895), britischer Biologe und Bildungsorganisator

\section{SENIOREN IN DEUTSCHLAND}

\section{Je älter, desto beschwerlicher}

In Deutschland fühlen sich drei Viertel der Menschen jenseits von 65+ fit und gesund. Unterschiede zwischen Männern und Frauen gibt es hier kaum. Dennoch: Im Alter wird es beschwerlicher. 17 Prozent der 65- bis 69-Jährigen fühlen sich gesundheitlich beeinträchtigt, in der Altersgruppe der ab 75-Jährigen sind es schon 29 Prozent.

Insgesamt sind ältere Menschen eher Sportmuffel. Wer sich dennoch bewegt, tut das am liebsten an der frischen Luft. Ein bisschen mehr davon würde jedoch nicht schaden, denn unter den 70- bis 74-Jährigen sind drei Viertel der Männer und fast zwei Drittel der Frauen übergewichtig. 